\title{
Dynamic Signature Recognition Based on Modified Windows Technique
}

\author{
Rafal Doroz and Krzysztof Wrobel \\ Institute of Computer Science, University of Silesia, Poland, \\ ul. Bedzinska 39, 41-200 Sosnowiec \\ \{rafal.doroz,krzysztof.wrobel\}@us.edu.pl
}

\begin{abstract}
The paper presents the method of signature recognition, which is a modification of the windows technique. This windows technique allows comparing signatures with the use of any similarity coefficient, without the necessity of using additional algorithms equalizing the lengths of the sequences being compared. The elaborated method introduces a modification regarding the repeatability of individual fragments of person's signature. Thus signature verification is performed only on the basis of signature fragments, characterized by the highest repeatability. Studies using the proposed modification have shown that it has a higher efficiency in comparison to the standard method.
\end{abstract}

Keywords: Signature recognition, windows technique, similarity measure.

\section{Introduction}

Biometric techniques are currently among the most dynamically developing areas of science. They prove their usefulness in the era of very high requirements set for security systems. Biometrics can be defined as a method of recognition and personal identification based on physical and behavioural features [1,4,5,12]. Physiological biometrics covers data coming directly from a measurement of a part of human body, e.g. a fingerprint, a shape of face, a retina. Behavioural biometrics analyses data obtained on the basis of an activity performed by a given person, e.g. speech, handwritten signature.

Data collection process within a signature recognition process can be divided into two categories: static and dynamic. The static system collects data using off-line devices [11]. A signature is put on paper, and then is converted into a digital form with the use of a scanner or a digital camera. In this case, the shape of the signature is the only data source, without the possibility of using dynamic data. On the other hand, dynamic systems use on-line devices, which register, apart from the image of the signature, also dynamic data connected with it. The most popular on-line devices are graphics tablets. Thanks to tablets, a signature can be recorded in the form of an $n$ point set $[5,6]$. Values of individual features such as: position, inclination, and pressure of a pen are determined in each point. Fig. 1 presents an example of signature $S_{i}$. 


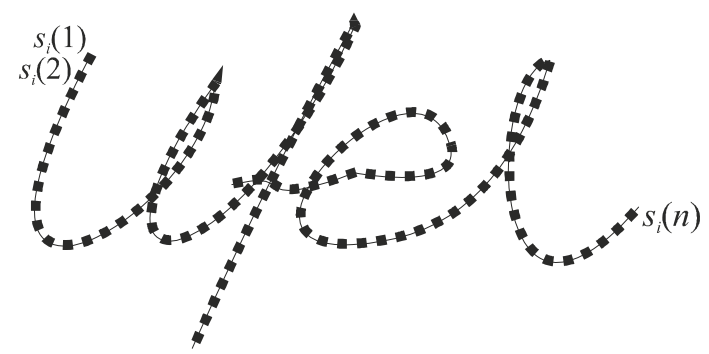

Fig. 1. Sample of signature $S_{i}$ and its selected points

When analysing signatures of the same person, it can be noticed that they differ from each other. Certain fragments of signatures are more similar to each other (repeatable) and other ones may differ considerably from one another. Example of such situation is presented in Fig. 2.

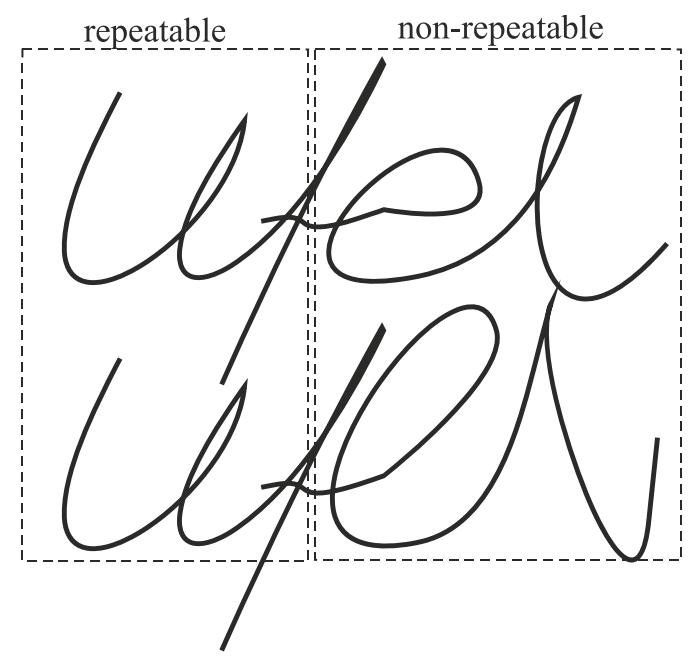

Fig. 2. Two signatures of the same person with a repeatable beginning

Many methods of determining the similarity between signatures require that the signatures being compared have the same length. This requirement is not always fulfilled, since the mentioned earlier differences between the signatures may result in their various lengths. This causes a necessity to use a method of equalizing the length of signatures.

Lengths of sequences can be equalized using many methods, such as DTW [16] or scaling methods [3]. A disadvantage of these methods is the need to interfere with the analysed data, which in turn may lead to distortion of the signatures being compared. The studies $[13,14,15]$ present a method, called windows technique, which allows determining the similarity of signatures without the necessity of initial equalization of their lengths. In this way, the signatures being compared are not distorted as it happened in the case of the DTW algorithm or the scaling method. 
The main goal on the investigation was to determine windows parameters to signature recognition level. The newest researches point out that new method of selection of some parameters gives better recognition level compare to previously reported work $[13,14,15]$. Between two compared signatures some differences can be observed, even for signatures of the same individual. It can also be observed that in many signatures some fragments are similar or not. For example one signer put signature almost the same at the beginning, while for other signer his signatures are very similar at the end. The main idea of the investigations is to find similarities and dissimilarities between fragments of the signatures. It will be more precisely explained in the next paragraphs of this paper.

\section{Window Technique}

In the windows technique, the $S_{i}$ and $S_{j}$ signatures being compared are divided into equal fragments. Each fragment contains $h$ signature points. Such fragments are called "windows" and are designated as "win". The $k$-th window in the $S_{i}$ signature is designated as $\operatorname{win} S_{i}(k)$, while $l$-th window in the $S_{j}$ signature - as $\operatorname{win} S_{j}(l)$. Next windows in the signature can be shifted in relation to each other by a certain number of points designated as jmp. In the $S_{i}$ signature this parameter was designated as jmp $S_{i}$, while in the $S_{j}$ signature - as $j m p S_{j}$. Appropriate selection of values of the jmp parameter affects the speed and effectiveness of the method.

The values of the $h$ and jmp parameters affect the number of the windows in the analysed signature. The number of all windows in the $S_{i}$ signature is designated as $n w S_{i}$, while in the $S_{j}$ signature - as $n w S_{j}$. The windows technique and the influence of parameters on the operation of this method have been discussed in detail in $[14,15]$. The division of the signature $S_{i}$ into windows is shown in Fig. 3.

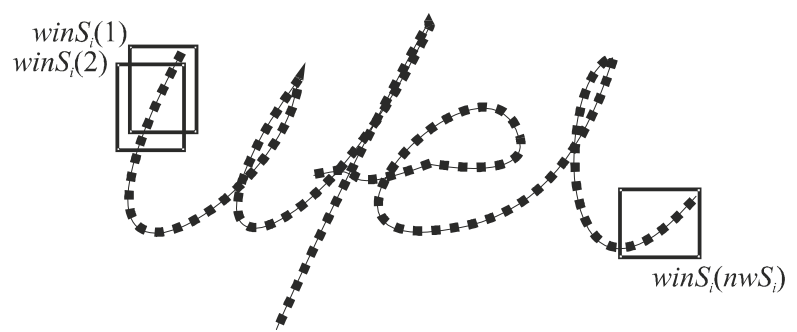

Fig. 3. Division of signature $S_{i}$ into windows, where $h=5, j m p S_{i}=1$

The process of comparing signatures consists in successive determination of the similarity between each window in the first signature and all windows in the second signature. An example illustrating a comparison of the first window in the $S_{i}$ signature with windows in the $S_{j}$ signature is shown in Fig. 4. 


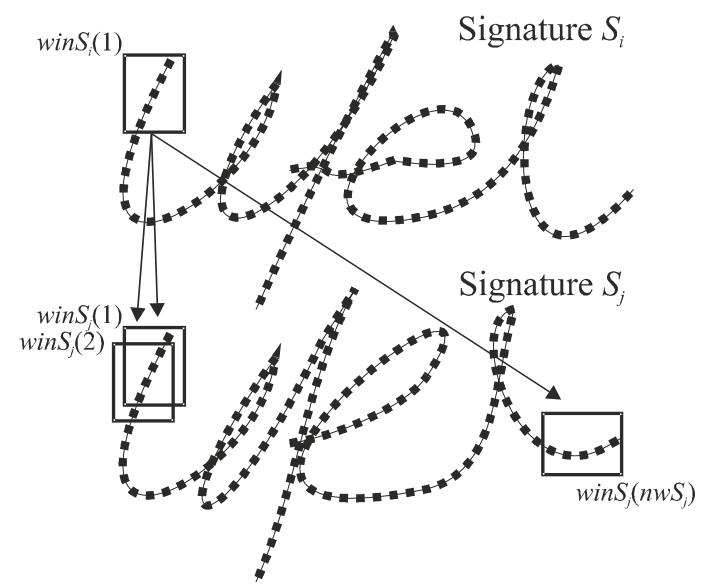

Fig. 4. Comparison of the windows in the two signatures

That same amount of data in the windows being compared allows using any similarity measure $M$. For each pair of the windows being compared, $\operatorname{win} S_{i}(k)$ in the $S_{i}$ signature and $\operatorname{win} S_{j}(l)$ in the $S_{j}$ signature, their similarity is calculated using the following formula:

$$
\operatorname{sim}_{k, l}=M\left(\operatorname{winS}_{i}(k), \operatorname{winS}_{j}(l)\right),
$$

where:

$M$ - similarity measure,

$k-$ number of the window in signature $S_{i}$,

$l \quad-$ number of the window in signature $S_{j}$.

The result of comparing the $k$-th window in the $S_{i}$ signature with the windows determined in the $S_{j}$ signature is the set of similarity values $S I M_{k}$ :

$$
\operatorname{SIM}_{k}=\left\{\operatorname{sim}_{k, 1}, \operatorname{sim}_{k, 2}, \ldots, \operatorname{sim}_{k, n w S_{j}}\right\},
$$

where:

$\operatorname{sim}_{k, l}$ - similarity between the compared windows $\operatorname{win} S_{i}(k)$ and $\operatorname{win} S_{j}(l)$, for $l=1, \ldots, n w S_{j}$.

The $\operatorname{SIM}_{k}$ set is determined for all windows created in the $S_{i}$ signature. After the similarity between all windows in the two signatures has been determined, the total similarity between the $S_{i}$ and $S_{j}$ signatures can be finally determined:

$$
W S\left(S_{i}, S_{j}\right)=\frac{1}{n w S_{i}} \sum_{k=1}^{n w S_{i}} \max \left(\operatorname{SIM}_{k}\right),
$$

where:

$W S\left(S_{i}, S_{j}\right) \quad-\quad$ similarity between the $S_{i}$ and $S_{j}$ signatures. 
The parameter in the windows technique, which affects the speed and effectiveness of the method, is dist. It narrows down the range of windows in the $S_{j}$ signature, with which the window analysed in the $S_{i}$ signature is being compared.

So far the value of the dist parameter has been the same for all the signatures of each person. A modification of the windows technique has been presented in this study, thanks to which the range of windows being compared is selected individually for signatures of each person. It allowed obtaining better results of the classification. The proposed modification is described in detail in the next section.

\section{Modification of the Window Technique}

The Tabdist arrays constitute a key element in the modification of the windows technique. The $P S$ set containing the genuine signatures of a given person is required for creating these arrays.

$$
P S=\left\{S_{1}, S_{2}, \ldots, S_{n s}\right\}
$$

where:

$n s$ - number of genuine signatures in $P S$ set,

$S_{i}-i$-th signature of the person, where $i=1, \ldots, n s$.

The Tabdist arrays are created separately for each genuine signature $S_{i} \in P S$. The $P S$ set must contain at least three signatures. However the number of elements in the $P S$ set should be as large as possible, because it is easier to assess the repeatability of signatures in a larger set.

The algorithm for determining the Tabdist array involves comparing the signatures from the $P S$ genuine set using the round robin method. The comparison is performed for the parameter values determined in the windows technique $\left(j m p S_{i}\right)$. The operation of the algorithm for determining the Tabdist array for the $S_{i} \in P S$ signature can be presented in several steps.

Step 1 - let $k=1$.

Step 2 - determine successively the similarity between the $\operatorname{win}_{i}(k)$ of the $S_{i}$ signature in relation to all windows created in the $S_{j} \in P S \backslash\left\{S_{i}\right\}$ signature, as shown in Fig. 4.

Step 3 - determine the $\operatorname{SIM}_{k}$ similarity set containing the results of comparisons between the $k$-th window of the $S_{i}$ signature and the windows created in the $S_{j}$ signature.

$$
\operatorname{SIM}_{k}=\left\{\operatorname{sim}_{k, 1}, \operatorname{sim}_{k, 2}, \ldots, \operatorname{sim}_{k, n w S_{j}}\right\}
$$

where:

$n w S_{j}-$ number of the window in signature $S_{j}$. 
Step 4 - determine the maximum value of the similarity from the $\operatorname{SIM}_{k}$ set and remember the number of the $m s_{k}$ window in the $S_{j}$ signature, for which this value was determined. This number can be determined from the formula:

$$
m s_{k}=\underset{i}{\arg \max }\left\{\operatorname{sim}_{k, i} \in S I M_{k}\right\}, \quad i=1, \ldots, n w S_{j} .
$$

Step 5 - determine the number of the $S_{j}$ signature point, in which the window with the $m s_{k}$ number begins:

$$
p m s_{k}=\left(\left(m s_{k}-1\right) \cdot j m p S_{j}\right)+1
$$

where:

$p m s_{k}-$ number of the signature point, in which $m s_{k}$ window begins.

Step 6 - normalize the $p m s_{k}$ value to the $[0,1]$ interval using the following formula:

$$
n m p s_{k}=\frac{p m s_{k}}{m}
$$

where:

$m \quad-$ number of $S_{j}$ signature points.

Step 7 - write the value of the $n m p s_{k}$ parameter in $k$-th column of the Tabdist array. A sample $n m p s_{k}$ value, for $k=1$, calculated when comparing the $S_{i}$ and $S_{j}$ signatures is presented in Table 1.

Table 1. Table Tabdist completed for first window in $S_{i}$ signature

\begin{tabular}{|c|c|c|c|c|c|}
\cline { 2 - 5 } \multicolumn{1}{c|}{} & $n m p s_{1}$ & $n m p s_{2}$ & $n m p s_{3}$ & $\ldots$ & $n m p s_{n w S_{i}}$ \\
\hline$S_{i} \leftrightarrow S_{j}$ & 0.018 & $\ldots$ & $\ldots$ & $\ldots$ & $\ldots$ \\
\hline
\end{tabular}

Step 8 - repeat steps 2 through 7 successively for $k=2, \ldots, n w S_{i}$. As a result of carrying out the steps 1 through 8 of the aforementioned algorithm, the first row of the Tabdist array is populated with values (Table 2).

Table 2. Table Tabdist completed for all windows in $S_{i}$ signature

\begin{tabular}{|c|c|c|c|c|c|}
\cline { 2 - 6 } \multicolumn{1}{c|}{} & $n m p s_{1}$ & $n m p s_{2}$ & $n m p s_{3}$ & $\ldots$ & $n m p s_{n w S_{i}}$ \\
\hline$S_{i} \leftrightarrow S_{j}$ & 0.018 & 0.027 & 0.036 & $\ldots$ & 0.943 \\
\hline
\end{tabular}

Step 9 - repeat the steps 1 through 8, comparing each time the $S_{i}$ signature with the next genuine signature from the $P S$ set. As a result of comparing each pair of the signatures the next row of the Tabdist array is obtained.

Sample Tabdist array for the $S_{1}$ signature from the $P S=\left\{S_{1}, S_{2}, S_{3}, S_{4}\right\}$ set is presented in Table 3. 
Table 3. Fragment of the table Tabdist completed for $S_{1}$ signature

\begin{tabular}{|c|c|c|c|c|c|}
\cline { 2 - 6 } \multicolumn{1}{c|}{} & $n m p s_{1}$ & $n m p s_{2}$ & $n m p s_{3}$ & $\ldots$ & $n m p s_{n w S_{1}}$ \\
\hline$S_{1} \leftrightarrow S_{2}$ & 0.018 & 0.027 & 0.036 & $\ldots$ & 0.943 \\
\hline$S_{1} \leftrightarrow S_{3}$ & 0.026 & 0.040 & 0.053 & $\ldots$ & 0.917 \\
\hline$S_{1} \leftrightarrow S_{4}$ & 0.013 & 0.026 & 0.039 & $\ldots$ & 0.918 \\
\hline
\end{tabular}

Step 10 - after the Tabdist array for the $S_{i}$ signature has been created, mean values $\overline{n m p s}$ and standard deviation values $\sigma$ for individual columns of the Tabdist array are determined (table 4).

Table 4. Fragment of the table Tabdist completed for $S_{1}$ signature

\begin{tabular}{|c|c|c|c|c|c|}
\cline { 2 - 6 } \multicolumn{1}{c|}{} & $n m p s_{1}$ & $n m p s_{2}$ & $n m p s_{3}$ & $\ldots$ & $n m p s_{n w S_{1}}$ \\
\hline$S_{1} \leftrightarrow S_{2}$ & 0.018 & 0.027 & 0.036 & $\ldots$ & 0.943 \\
\hline$S_{1} \leftrightarrow S_{3}$ & 0.026 & 0.040 & 0.053 & $\ldots$ & 0.917 \\
\hline$S_{1} \leftrightarrow S_{4}$ & 0.013 & 0.026 & 0.039 & $\ldots$ & 0.918 \\
\hline$\overline{n m p s}$ & 0.019 & 0.031 & 0.043 & $\ldots$ & 0.926 \\
\hline$\sigma$ & 0.006 & 0.008 & 0.009 & $\ldots$ & 0.015 \\
\hline
\end{tabular}

Step 11 - remove from the Tabdist array the columns, in which the standard deviation value is greater than a certain threshold value $\zeta \in[0,1]$. Removing these columns from the Tabdist array causes that the non-repeatable signature fragments are not compared with each other.

Thanks to the Tabdist array the $k$-th window in the $S_{i}$ genuine signature is compared with a sequence of windows in the $S_{j}$ signature. The number of the first and the last window in the sequence is determined using the following formulas:

$$
\begin{aligned}
& p w i n(k)=\operatorname{round}(\overline{n p m s}(k) \cdot m), \text { for } k=1, \ldots, n w S_{i}, \\
& p_{w i n}(k)=\operatorname{round}(p \operatorname{win}(k)-\sigma(k) \cdot m), \text { for } k=1, \ldots, n w S_{i}, \\
& \text { pwin }_{\max }(k)=\operatorname{round}(p \operatorname{win}(k)+\sigma(k) \cdot m), \text { for } k=1, \ldots, n w S_{i} .
\end{aligned}
$$

where:

$$
\begin{aligned}
& \operatorname{pwin}(k) \quad-\quad \text { the middle window in the sequence, } \\
& \operatorname{pwin}_{\min }(k) \quad-\quad \text { the first window in the sequence, } \\
& \operatorname{pwin}_{\max }(k) \quad-\quad \text { the last window in the sequence, } \\
& \overline{n p m s}(k) \quad-\quad \text { the mean value read from the } k \text {-th column of the Tabdist array for } \\
& k \text {-th window in the } S_{i} \text { signature, } \\
& \sigma(k) \quad-\quad \text { the standard deviation read from the } k \text {-th column of the Tabdist } \\
& \text { array for } k \text {-th window in the } S_{i} \text { signature, } \\
& m \quad-\quad \text { number of the } S_{j} \text { signature point, } \\
& n w S_{i} \quad-\quad \text { number of the window in signature } S_{i} \text {. }
\end{aligned}
$$


Figure 5 shows the manner of comparing the windows, taking into account the Tabdist array.

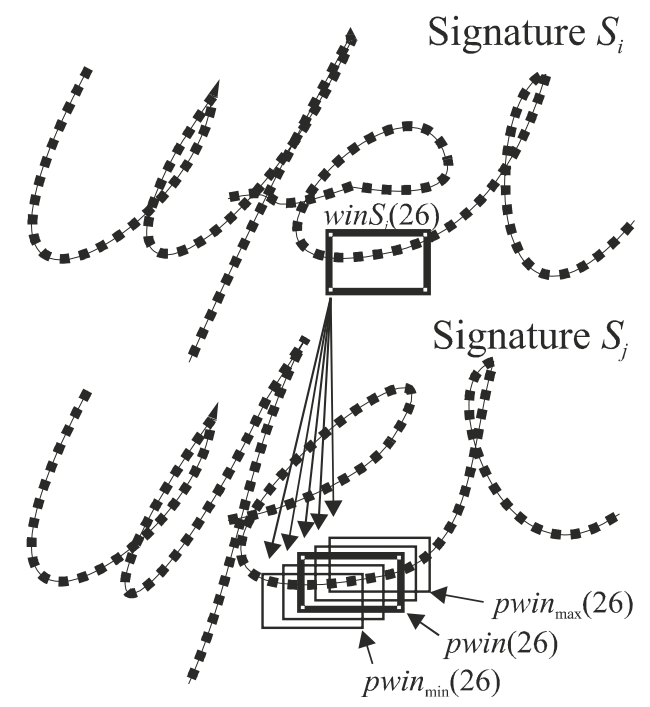

Fig. 5. The example of comparing the windows, taking into account the Tabdist

Step 12 - end the algorithm, if the $S_{i}$ signature has been compared with all the signatures from the $P S$ set.

The result of the operation of the modified windows technique is the Tabdist array generated for each genuine signature. This array is then used in the signature comparison process.

\section{$4 \quad$ Research Results}

The purpose of the studies was to determine the effectiveness of the proposed modification and its impact on the signature verification results. The standard windows method was compared with the modified method in the course of the studies. The studies were conducted with the use of signatures from the MCYT database [20]. The database used in the studies contained 1000 signatures of 100 persons. A recognized signature belonging to a given person was compared with 5 genuine signatures of the same person. The set of genuine signatures did not contain any recognizable signatures. The recognizable signatures included 3 other original signatures of a given person and 2 forged signatures of this person.

The studies were conducted for the following ranges of parameter values:

- dist $=[0.1, \ldots, 0.5]$ with a step of 0.1 ,

- $h=[10, \ldots, 50]$ with a step of 10 ,

- the standard deviation $\sigma=[0.05, \ldots, 0.2]$ with a step of 0.05 . 
All combinations of the above parameters were examined. During the research, the several similarity coefficients have been used $[14,15]$. The best results were obtained with the use of $\mathrm{R}^{2}$ ratio [1]. During the studies, the following signature features were examined: coordinates $(x, y)$ of signature points, pen pressure $p$ at the point $(x, y)$. EER was calculated for each measurement. The lower the value of EER, the lower error of a given measurement is. Table 5 shows the results of the studies obtained for the standard and modified windows method.

Table 5. The best measurements results for standard and modified window technique

\begin{tabular}{|l|c|c|c|}
\cline { 2 - 4 } \multicolumn{1}{c|}{} & $\begin{array}{c}\text { Number of } \\
\text { points in } \\
\text { window } h\end{array}$ & $\begin{array}{c}\text { Standard } \\
\text { deviation } \\
\text { values } \sigma\end{array}$ & EER [\%] \\
\hline Standard window technique & 40 & 0.3 & 6.59 \\
\hline Modified window technique & 40 & 0.2 & 3.20 \\
\hline
\end{tabular}

\section{Conclusions}

The use of the modified windows technique allows to reduce verification error rate in comparison to standard windows technique. The obtained result EER $=3.20 \%$ is also competitive in comparison to other methods, known from the literature. Table 6 summarizes the well-known methods of signature recognition published in recent years. The methods shown in Table 6 were tested by their authors, as in the present study, using the MCYT signature database.

Table 6. Different online signature verification methods

\begin{tabular}{|l|c|}
\hline \multicolumn{1}{|c|}{ Authors } & Results (EER [\%]) \\
\hline Presented method & $\mathbf{3 . 2 0}$ \\
\hline Fierrez J., Ortega - Garcia J., Ramos D., Gonzalez - Rodriguez J. [2] & 0.74 \\
\hline Lumini A., Nanni L. [7] & 4.50 \\
\hline Maiorana E. [8] & 8.33 \\
\hline Nanni L., Lumini A. [9] & 21.00 \\
\hline Nanni L., Maiorana E., Lumini A., Campisi P. [10] & 3.00 \\
\hline Vargas J. F., Ferrer M. A., Travieso C. M., Alonso J. B. [17] & 12.82 \\
\hline Vivaracho - Pascual C., Faundez - Zanuy M., Pascual J. M. [18] & 1.80 \\
\hline Wen J., Fang B., Tang Y. Y., Zhang T. [19] & 15.30 \\
\hline
\end{tabular}

The important advantages the proposed method is to determine of the parameter dist. It should be noted that this parameter is automatically selected. This selection is possible on the basis of analyzing of the Tabdist array. The obtained classification results encourage the further modification of the presented technique. In the next investigations stages using more complex methods of data analysis are planned. Additionally, time and memory complexity will be also estimated. 


\section{References}

1. Doroz, R., Porwik, P., Para, T., Wróbel, K.: Dynamic Signature Recognition Based on Velocity Changes of Some Features. International Journal of Biometrics 1(1), 47-62 (2008)

2. Fierrez, J., Ortega-Garcia, J., Ramos, D., Gonzalez-Rodriguez, J.: HMM-based On-line Signature Verification: Feature Extraction and Signature Modeling. Pattern Recognition Letters 28(16), 2325-2334 (2007)

3. Foley, J.D.: Introduction to Computer Graphics. Addison-Wesley (1993)

4. Impedovo, S., Pirlo, G.: Verification of Handwritten Signatures: an Overview. In: 14th International Conference on Image Analysis and Processing, pp. 191-196 (2007)

5. Kamel, M.S., Ellis, G.A., Sayeed, S.: Glove-based Approach to Online Signature Verification. IEEE Transactions on Pattern Analysis and Machine Intelligence, 1109-1113 (2008)

6. Khan, M.K., Khan, M.A., Khan, M.A.U., Ahmad, I.: On-Line Signature Verification by Exploiting Inter-Feature Dependencies. In: 18th International Conference on Pattern Recognition, pp. 796-799 (2006)

7. Lumini, A., Nanni, L.: Ensemble of On-line Signature Matchers Based on Over Complete Feature Generation. Expert Systems with Applications 36(3), 5291-5296 (2009)

8. Maiorana, E.: Biometric Cryptosystem Using Function Based On-line Signature Recognition. Expert Systems with Applications 37(4), 3454-3461 (2010)

9. Nanni, L., Lumini, A.: Ensemble of Parzen Window Classifiers for On-line Signature verification. Neurocomputing 68, 217-224 (2005)

10. Nanni, L., Maiorana, E., Lumini, A., Campisi, P.: Combining local, regional and global matchers for a template protected on-line signature verification system. Expert Systems with Applications 37(5), 3676-3684 (2010)

11. Porwik, P.: The Compact Three Stages Method of the Signature Recognition. In: 6th IEEE International Conference on Computer Information Systems and Industrial Management Applications, pp. 282-287 (2007)

12. Porwik, P., Para, T.: Some Handwritten Signature Parameters in Biometric Recognition Process. In: 29th IEEE International Conference on Information Technology Interfaces, pp. 185-190 (2007)

13. Porwik, P., Doroz, R., Wrobel, K.: A New Signature Similarity Measure. In: 8th IEEE Int. Conf. on Computer Information Systems and Industrial Management Applications, Coimbatore, India, pp. 1022-1027 (2009)

14. Porwik, P., Doroz, R., Wróbel, K.: A New Signature Similarity Measure Based on Windows Allocation Technique. International Journal of Computer Information Systems and Industrial Management Applications (IJCISIM) 2, 297-305 (2010)

15. Porwik, P., Wrobel, K., Doroz, R.: Signature Recognition Method by Means of the Windows Technique. An International Journal of Image Processing \& Communications 14(2-3), 43-50 (2009)

16. Shanker, A.P., Rajagopalan, A.N.: Off-line signature verification using DTW. Pattern Recognition Letters 28, 1407-1414 (2007)

17. Vargas, J.F., Ferrer, M.A., Travieso, C.M., Alonso, J.B.: Off-line Signature Verification Based on Grey Level Information Using Texture Features. Pattern Recognition 44(2), 375-385 (2011)

18. Vivaracho-Pascual, C., Faundez-Zanuy, M., Pascual, J.M.: An Efficient Low Cost Approach for On-line Signature Recognition Based on Length Normalization and Fractional Distances. Pattern Recognition 42(1), 183-193 (2009)

19. Wen, J., Fang, B., Tang, Y.Y., Zhang, T.: Model-based Signature Verification with Rotation Invariant Features. Pattern Recognition 42(7), 1458-1466 (2009)

20. http://atvs.ii.uam.es/databases.j.sp 